\title{
Measurement of the temperature-dependent output of lead zirconate titanate transducers
}

\author{
Marina Bakaric ${ }^{\mathrm{a}, \mathrm{b}, *}$, Paul Fromme ${ }^{\mathrm{c}}$, Andrew Hurrell ${ }^{\mathrm{d}}$, Srinath Rajagopal ${ }^{\mathrm{b}}$, \\ Piero Miloro ${ }^{\mathrm{b}}$, Bajram Zeqiri ${ }^{\mathrm{a}} \mathrm{b}$, Benjamin T. Cox ${ }^{\mathrm{a}}$, Bradley E. Treeby ${ }^{\mathrm{a}}$ \\ ${ }^{a}$ Department of Medical Physics and Biomedical Engineering, University College London, \\ London, UK \\ ${ }^{b}$ Centre for Chemical, Environmental and Medical Science, National Physical Laboratory, \\ Teddington, UK \\ ${ }^{c}$ Department of Mechanical Engineering, University College London, London, UK \\ ${ }^{d}$ Precision Acoustics Ltd., Higher Bockhampton, Dorchester, UK
}

\begin{abstract}
The effect of temperature and electrical drive conditions on the output of lead zirconate titanate (PZT) transducers is of particular interest in ultrasound metrology and medical ultrasound applications. In this work, the temperaturedependent output of two single-element PZT transducers was measured between $22{ }^{\circ} \mathrm{C}$ and $46{ }^{\circ} \mathrm{C}$. Two independent measurement methods were used, namely radiation force balance measurements and laser vibrometry. When driven at constant voltage using a $50 \Omega$ matched signal generator and amplifier using continuous wave $(\mathrm{CW})$ or quasi- $\mathrm{CW}$ excitation, the output of the two transducers increased on average by $0.6 \%$ per degree, largely due to an increase in transducer efficiency with temperature. The two measurement methods showed close agreement. Similar trends were observed when using single cycle excitation with the same signal chain. However, when driven using a pulser (which is not electrically matched), the two transducers exhibited different behaviour depending on their electrical impedance. Accounting for the temperature-dependent output of PZT transducers could have implications for many areas of ultrasound metrology, for example, in therapeutic ultrasound where a coupling fluid at an
\end{abstract}

\footnotetext{
${ }^{*}$ Corresponding author: Marina Bakaric, Department of Medical Physics and Biomedical Engineering, University College London, Malet Place Engineering, Gower Street, London WC1E 6BT, UK

Email address: marina.bakaric.16@ucl.ac.uk (Marina Bakaric)
} 
increased or decreased temperature is often used.

Keywords: laser vibrometry, metrology, PZT, radiation force balance, transducer, ultrasound

\section{Introduction}

\subsection{Overview}

Accurate knowledge of the output of ultrasound transducers output is essential in ultrasound metrology, as well as in medical ultrasound applications in

5 order to ensure safety during both imaging and therapy. For example, if an array of sources is to be used for applications such as transcranial ultrasonic neuromodulation [1, their output must be validated to ensure that the amplitude of acoustic pressure can be estimated at the target location, thereby contributing to the safety and effectiveness of the delivered treatment 2. This validation is usually done experimentally, using techniques such as acoustic holography [3 where a calibrated hydrophone is scanned through the acoustic field of a source transducer in order to measure the generated ultrasound pressure field.

Hydrophones are required for absolute measurements of the properties of an acoustic field and their sensitivity is calibrated according to the IEC 62127-2 4] standard. A companion standard also specifies the methods for measurements of acoustic fields generated by ultrasound medical equipment in liquids (IEC 62127-1 [5]). During these measurements, the environment (i.e. liquid) temperature should ideally match the temperature at which the hydrophone was calibrated. The electroacoustic properties of hydrophones vary with ambient temperature [6] and for this reason, they are usually calibrated close to the intended application temperature. For most applications, the calibration is performed at a room temperature of $22{ }^{\circ} \mathrm{C} \pm 3{ }^{\circ} \mathrm{C}$ [7. Although corrections to hydrophone sensitivity and frequency response values may be applied in order to adjust the derived transducer characteristics to a different temperature of interest, these corrections are only known for a small range of temperatures. 
Additional tests must also be undertaken to show the results comply with the standards 7 .

A problem potentially arises when the transducer is then used in an application in which the temperature is different from the calibration temperature. An

30 example of this is again transcranial ultrasonic therapy, where the water used as a coupling agent between the transducer and the subject can be either cooled to reduce skin burns or warmed closer to body temperature to improve comfort. In this case, the transducers may operate at a temperature approximately $5{ }^{\circ} \mathrm{C}$ below [8] or up to $15^{\circ} \mathrm{C}$ above the characterisation temperature, and as such, their output is likely to vary. Additionally, transducer output can change due to inefficiencies in transduction, where self-heating occurs. Thus a suitable method must be used in order to investigate the variability of transducer output with temperature.

\subsection{PZT transducers}

Piezoelectric ultrasound transducers use the inverse piezoelectric effect to generate ultrasound waves. A piezoelectric ceramic that is frequently used in medical ultrasound is lead zirconate titanate (PZT). Piezoelectric materials and their electromechanical properties can be fully characterised with a set of independent mechanical, piezoelectric and electrical parameters [9]. The piezoelectric coupling factor $k$ can then be calculated, which is a measure of the efficiency with which the crystal converts energy from electrical to mechanical. Note, however, that this does not take into account losses in the system and is therefore not directly equivalent to transducer efficiency [10]. The piezoelectric coupling factor depends on the boundary conditions surrounding the element and the piezoelectric element vibration mode. In the case of a PZT transducer operating in thickness mode, with lateral dimensions much greater than the thickness of the piezoelectric layer, the two most relevant piezoelectric coupling factors are defined as [9]:

$$
k_{33}^{t}=\frac{e_{33}}{\sqrt{c_{33}^{D} \epsilon_{33}^{S}}}, k_{33}^{l}=\frac{d_{33}}{s_{33}^{E} \epsilon_{33}^{T}}
$$


where $k_{33}^{t}$ represents lateral clamping and $k_{33}^{l}$ strain-free (unclamped) conditions $\left(k_{33}^{t}<k_{33}^{l}\right)$. The parameters in Eq. 1 are defined in Table 1 . Of particular

Table 1: Mechanical and piezoelectric coefficients used in the calculation of the piezoelectric coupling factors 9 .

\begin{tabular}{llll}
\hline Coefficient & Description & SI Units & Constant \\
\hline$e_{31}, e_{33}$ & piezoelectric stress & $\mathrm{C} / \mathrm{m}^{2}$ & \\
& coefficient & & \\
$d_{31}, d_{33}$ & piezoelectric coefficient & $\mathrm{C} / \mathrm{N}$ & \\
$c_{33}^{D}$ & elastic stiffness & $\mathrm{N} / \mathrm{m}^{2}$ & dielectric displacement $D$ \\
$\epsilon_{31}^{T}, \epsilon_{33}^{S}, \epsilon_{33}^{T}$ & dielectric permittivity & $\mathrm{F} / \mathrm{m}$ & strain $S$, stress $T$ \\
$s_{11}^{E}, s_{33}^{E}$ & elastic compliance & $\mathrm{m}^{2} / \mathrm{N}$ & electric field $E$ \\
\hline
\end{tabular}

interest here are the transmission (strain) coefficient $d$ describing the strain produced per unit of applied electric field, and parameter $e$ relating the mechanical and electrical properties of the material. Additionally, a change in 45 dielectric properties alters the capacitance of a piezoelectric material and thus the electrical matching criteria of a PZT transducer.

The temperature dependence of the elastic, dielectric and piezoelectric coefficients of PZT ceramics and films has been investigated in several studies, with measurements performed on a variety of PZT types ranging from commercially available PZT-5H [11, various hard and soft PZT materials such as PZT 52/48 and PZT-500 [12], respectively, to PZT mixtures with varying concentrations of $\mathrm{PbTiO}_{3}[13$. 1 All studies reported an increase in transmission coefficients

\footnotetext{
${ }^{1}$ The designation of soft and hard PZT ceramics refers to the mobility of dipoles (or domains) and hence also to the polarization and depolarization behavior. Briefly, soft ceramics are created by adding small amounts of a donor dopant leading to a creation of metal (cation) vacancies, while hard PZTs are doped with acceptor ions thus creating oxygen (anion) vacancies in the crystal structure [14. These dopants in turn determine the characteristic features of the PZT material and thus its application suitability.
} 
$d_{31}$ and $d_{33}$ with temperature [11, 12, 13, 15, 16, 17] as well as $e_{31}$ [13, where a stronger dependency was associated with a decreasing $\mathrm{PbTiO}_{3}$ content. The relative dielectric permittivities $\epsilon_{11}^{T}$ and $\epsilon_{33}^{T}$ exhibited a very large increase with temperature [11, 12, 13, 17, 18, while elastic compliance coefficients $s_{11}^{E}$ and $s_{33}^{E}$ followed a weakly increasing trend for soft PZT, but no change was observed in hard PZT materials [17. The piezoelectric coupling factors $k_{11}$ and $k_{33}$ increased for undoped PZT [15], while $k_{33}^{t}$ was independent of temperature in

${ }_{60} \quad$ a study that also showed $k_{33}^{l}$ decreases for hard PZT whereas for soft PZT it increases until $0{ }^{\circ} \mathrm{C}$ followed by a decreasing trend [17. In summary, changes in temperature have greater influence on the properties of soft PZT materials [17. than hard PZTs, and their response depends on dopants present [19.

Using the existing literature, it is challenging to predict the precise behaviour 65 of a PZT transducer at elevated temperatures. The properties of the material used for the piezoelectric layer have a strong dependence on dopants as well as the field conditions such as temperature and hydrostatic pressure. These coefficients also do not represent all the factors influencing the ultrasound transducer output. For example, the electrical impedance of the transducer and its matching with the signal chain must also be taken into consideration.

\subsection{Paper Outline}

In this paper, two independent methods are used to investigate the effect of temperature increase on the output of two hard PZT ceramic transducers. The first method uses radiation force balances as a recommended technique for determination of transducer power output as given in IEC 61161 [20]. The second method, laser vibrometry, is based on optical interferometry, where the sound pressure is calculated from the measurement of the displacement of an acoustically reflective membrane (IEC 62127-2 [4]).

The effect of excitation source type on the temperature-dependent transducer output is investigated using laser vibrometry and electrical impedance measurements. Three driving conditions are assessed: an impedance-matched signal generator and amplifier using narrowband (1) single cycle or (2) long 
burst excitation, and (3) a pulser providing a source for broadband excitation.

The methods used are described in Section 2, followed by details on data analysis and measurement uncertainty calculations. The study findings are presented in Section 3 and conclusions derived thereof.

\section{Methods}

\subsection{Radiation force balance}

\subsubsection{Theoretical background}

The propagation of ultrasound through a medium leads to a simultaneous transfer of momentum. If the ultrasound beam is intercepted by a target, the resulting radiation force on it is proportional to the acoustic power $W_{t o t}[21,22$. This principle is exploited by radiation force balances (RFB), internationally accepted for characterizing ultrasound power [20]. Radiation force $F$ is typically determined by measuring the change in weight $\Delta m$ of an initially buoyant target 23]:

$$
F=\Delta m g
$$

where $g$ is the acceleration due to gravity. For a plane wave incident on a perfectly absorbing target, the radiation force is given by:

$$
F=\frac{W_{t o t}}{c_{w}}
$$

where $W_{\text {tot }}$ is the total acoustic power emitted by the transducer and $c_{w}$ is the speed of sound of the medium, usually water [24]. For a reflective target, the force depends on the target geometry and material, and Eq. 3 can be generalised by multiplying by the right hand side by the factor $h$

$$
h=1+R \cos 2 \theta
$$

90

where $R$ is the amplitude reflectivity coefficient of the target surface, and $\theta$ is the angle between the direction of the incident ultrasound beam and the normal to the target surface 21. For absorbing targets no correction is needed. 
A typical RFB setup can be seen in Fig. 1. The transducer-target separation $x$ in the setup is determined by the target type, but is usually kept at the minimum distance possible in order to reduce the effects of attenuation and streaming [21. Due to the acoustic absorption of ultrasound in water, the power loss from the beam increases with the distance and a correction for this is applied in the form:

$$
W_{\text {corr }}=W_{t o t} e^{2 \alpha x}
$$

where $W_{\text {corr }}$ is the corrected transducer power output, $W_{\text {tot }}$ is the measured power, and $\alpha$ is the absorption coefficient in $\mathrm{Np} / \mathrm{m}$ at the measurement frequency. Under a plane wave assumption and using the expression for the average acoustic intensity [9], the acoustic pressure $p$ generated by the transducer is proportional to:

$$
p \propto \sqrt{\rho_{w} c_{w} W_{c o r r}}
$$

where $\rho_{w}$ and $c_{w}$ are the temperature-dependent density and speed of sound in water, respectively [25, 26].

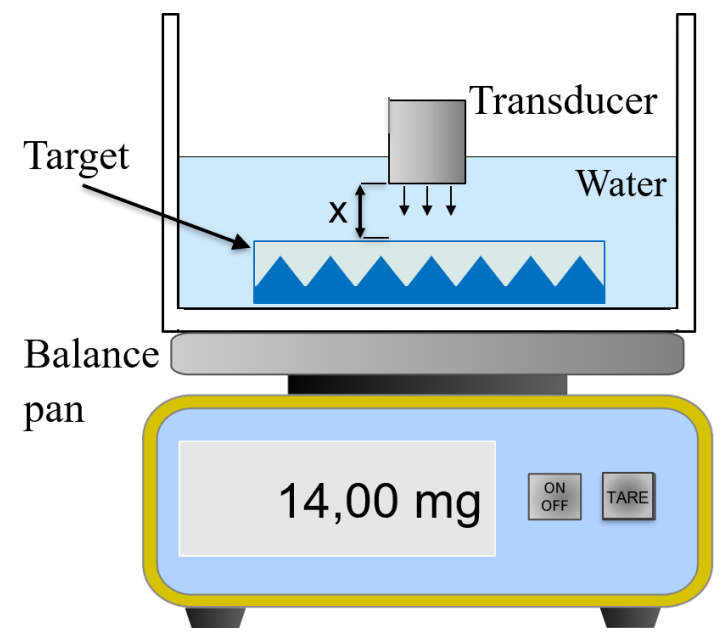

Figure 1: Radiation force balance setup. Transducer is positioned above the suspended target immersed in a tank filled with degassed deionised water, with the transducer's beam axis perpendicular to the target. The tank is placed on the balance pan and the balance readout sent to a PC so as to track the changes in weight caused by the ultrasound transmitted from the transducer. 


\subsubsection{Measurement setup}

The transducers used in this study were two unfocussed PZT transducers from Olympus (Panametrics NDT, Tokyo, Japan), namely a $1 \mathrm{MHz}$ transducer (A392S-SU, U8421057) with an active diameter of 1.5" (38.1 mm) and a $2.25 \mathrm{MHz}$ transducer (A304S-SU, U8421006) with an active diameter of 1" (25.4 mm). The characterisation of the transducer output power was performed using the radiation force balance configurations available at the National Physical Laboratory (Teddington, UK), both utilising a high-performance analytical balance (AC211S Balance, Sartorius, Göttingen, Germany). Two configurations were chosen, shown in Fig. 2. The schematic of the two balance configurations in Fig. 2 can be found in IEC 61161, page 43, Figure F.2 and Figure F.3 [20]. These configurations are used to offset the weight of the water tank for top-pan load limitations of the balance. The frame holding the target is connected to the force measuring point on the balance and hence the weight of the tank is not felt by the balance.

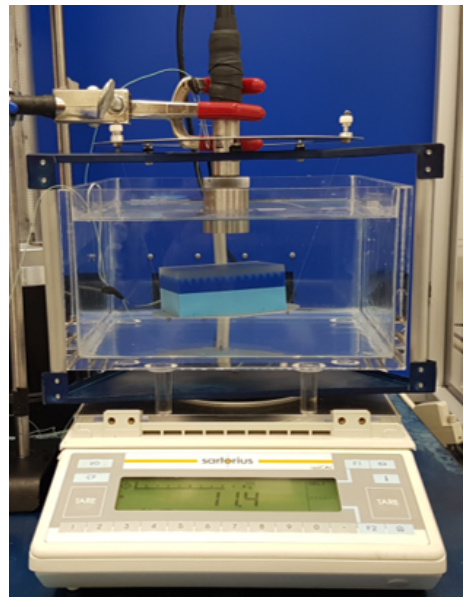

(a)

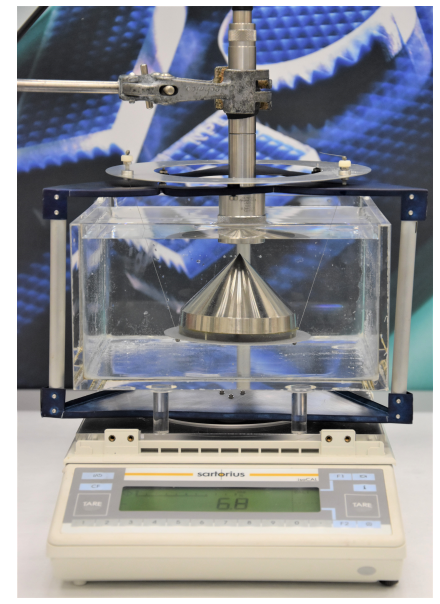

(b)

Figure 2: Radiation force balance (RFB) configurations with a suspended (a) flat absorbing target; (b) conical reflecting target.

Configuration 2(a) uses a suspended flat absorbing target made of either HAM A-LF (NPL, Teddington, UK) [27] as shown in Fig. 2(a) or Aptflex F28P 
(Precision Acoustics Ltd., Dorchester, UK) acoustic absorber material, both of which satisfy the requirements of IEC 61161 [20]. The advantages of using a flat absorbing target include simplified corrections for non-perpendicular incidence, as the radiation force is in this case insensitive to small changes in angular alignment [28, and the reduced distance between the transducer and the target, thereby reducing the magnitude of the applied corrections for small-signal attenuation in water, which can be significant at higher frequencies. However, the absorbed ultrasound will cause the target material to heat up, resulting in thermal expansion and a change in buoyancy. This in turn may cause the weight of the target to drift and give rise to errors in the measurement of the radiation force $[28$.

In configuration 2(b), a suspended air-backed convex cone is used as a reflecting target. In this case, the factor $h$ (Eq. 4) is determined by the direction and magnitude of the reflected beam assuming $100 \%$ reflectivity. The target used in 2(b) is right-angled ensuring the reflected ultrasound is directed perpendicular to the beam axis and thus away from the transducer. The main advantage of using a reflecting target is that it absorbs very little acoustic energy, so buoyancy changes due to thermal expansion of the target are negligible. Hence thermal drifts caused by absorption have no effect on the force being measured.

All measurements were performed in an enclosure with air temperature control. The tank was filled with degassed deionised water in order to prevent the occurrence of cavitation which can produce an increase in the attenuation of ultrasound due to scattering and absorption 29. The temperature of the air and water were monitored using K-type thermocouples and logged using a Pico thermocouple data logger (TC-08, Pico Technology, St Neots, UK). The water was preheated to $50{ }^{\circ} \mathrm{C}$, and measurements taken every $2{ }^{\circ} \mathrm{C}$ from $46{ }^{\circ} \mathrm{C}$ to room temperature of $22{ }^{\circ} \mathrm{C}$, at the state of thermal equilibrium, i.e. once both the enclosure air and water temperature were equal.

The PZT transducers were driven by a waveform generator (33600A, Keysight Technologies, Santa Rosa, CA, USA) connected via a $150 \mathrm{~W}$ power amplifier 
(150A100B, AR, Souderton, PA, USA), both matched to an electric impedance of $50 \Omega$. Measurements were made under continuous wave $(\mathrm{CW})$ conditions, where the transducer was excited by a sinusoidal wave with a frequency set to the centre frequency of the transducer. The transducer drive voltage was monitored using a Tektronix TPP0850 scope probe and MSO54 Tektronix 5 series mixed signal oscilloscope (Tektronix, U.K. Ltd., Berkshire, UK) and was kept constant at a peak positive voltage of $14 \mathrm{~V}$. This was done by adjusting the amplitude on the signal generator from $86 \mathrm{mVpp}$ at a temperature of $46{ }^{\circ} \mathrm{C}$ to $80 \mathrm{mVpp}$ at $22{ }^{\circ} \mathrm{C}$ for the $1 \mathrm{MHz}$ transducer, and from $72 \mathrm{mVpp}$ at a temperature of $46{ }^{\circ} \mathrm{C}$ to $66 \mathrm{mVpp}$ at $22{ }^{\circ} \mathrm{C}$ for the $2.25 \mathrm{MHz}$ transducer. Ideally, these measurements should be performed by keeping the input electrical power to the transducer constant. However, this requires knowledge of the temperature-dependent electrical impedance of the transducer, a parameter which is not always readily available. Instead, the drive voltage was kept constant throughout the RFB measurements as this parameter can be easily monitored and controlled during experiments 30 . The temperature-dependent electrical impedance was later measured to give the values of the electrical power input and the transducer efficiency, as described in Section 2.3 .

During the RFB measurements, the transducer was switched on for a period of $10 \mathrm{~s}$ and then switched off for a period of another $10 \mathrm{~s}$, with four on-off transitions for each measurement temperature. This procedure is known to improve the measurement quality, as well as enable the detection of any issues 165 related to the transducer output such as instability or self-heating 31. Data was acquired and processed by a LabVIEW application written in-house that accounts for the target type, temperature-dependent speed of sound of water, performs attenuation corrections given the transducer-target separation distance and minimises errors arising from drifts in "zero" levels and readout levels of the balance by extrapolating the change in readings to a common time in the measurement sequence. 


\subsection{Laser vibrometry}

\subsubsection{Theoretical background}

Heterodyne vibrometers are used in the primary calibration of ultrasound hydrophones, reconstructing the displacement signal from the phase modulation of the laser beam reflected from a vibrating surface using a suitable decoder [4, 32, 33. Here, the acoustic pressure generated by a transducer is determined by directing the ultrasound waves towards a thin reflective pellicle placed within a water vessel. A thin, acoustically transparent membrane follows the movement of the surrounding medium and can therefore reveal the particle displacement induced by an ultrasound field 23. Provided the pellicle is optically reflective, its displacement can be directly measured using optical interferometry [4]. As ultrasound waves are reflected at all boundaries between the water, pellicle and air multiple times, a model for the acoustic transmission coefficient $T$ of the displacement through the pellicle must be used [4,34. Assuming a plane wave, the acoustic pressure $p$ generated by an ultrasound transducer is related to the time derivative of the time-dependent acoustic displacement $\xi(t)$. Taking into account the acoustic absorption of water, the acoustic pressure inside the water can be calculated as:

$$
p=\frac{\rho_{w} c_{w}}{T} \frac{\partial \xi}{\partial t} e^{\alpha x}
$$

where $\rho_{w}$ and $c_{w}$ are the temperature-dependent density [25] and speed of sound

[26] of water, respectively, $T$ is the transmission factor of the pellicle while $\alpha$ is the absorption coefficient in $\mathrm{Np} / \mathrm{m}$ at the measurement frequency and $x$ is the transducer-pellicle distance in meters.

In the quasi-continuous excitation case, where the ultrasound waves have a narrowband frequency content centred around the transducer's centre frequency, the particle and thus pellicle displacement can be described with:

$$
\xi(t)=D e^{i \omega t}
$$

where $D$ is the amplitude of the pellicle displacement and $\omega$ is the angular frequency. In this case, the magnitude of the acoustic pressure generated by the 
transducer can be obtained as [4]:

$$
p=\frac{\rho_{w} c_{w} \omega D}{T} e^{\alpha x} .
$$

\subsubsection{Measurement setup}

A schematic of the measurement setup is shown in Fig. 3(a). The transducer under test was immersed in a tank filled with degassed deionised water, the temperature of which was controlled using a thermostat (ECO RE415S Silver thermostat, Lauda GmbH, Lauda-Königshofen, Germany) with an in-built PID controller and J-type thermocouple for feedback. The tank was lined with absorbers to avoid reflections and the generation of standing waves. The transducer was positioned with its front face parallel to the water surface. A thin reflective aluminium pellicle was placed on the water surface. This was done in order to avoid errors in the displacement measurements caused by the water surface vibrations arising from environmental sources as well as to increase measurement SNR through higher reflectivity. The pellicle transmission factor was calculated as described in [35] and was equal to $T=2$. The pellicle thickness was measured with a micrometer and was $20 \pm 2 \mu \mathrm{m}$. The pellicle was stretched across a holder design based on embroidery rings, $80 \mathrm{~mm}$ in diameter and positioned approximately $10 \mathrm{~mm}$ from the transducer face. The exact distance from the transducer was determined from the time of arrival of the signals and the known speed of sound in water [26].

The pellicle displacement caused by the ultrasound waves emitted from the transducer was interrogated using a laser vibrometer (Polytec OFV-5000 vibrometer and OFV-505 laser, Waldbronn, Germany). This was positioned on a 2-axis motorised stage above the tank, with its beam $(\lambda=633 \mathrm{~nm})$ directed perpendicular to the pellicle using a mirror (BB1-E02P, Thorlabs, Inc., Newton, NJ, USA). The vibrometer was coupled with a displacement decoder (Polytec DD-300, Waldbronn, Germany) with a scaling factor of $50 \mathrm{~nm} / \mathrm{V}$. Signals were filtered using a Butterworth high-pass filter with a cutoff frequency of $0.5 \mathrm{MHz}$, and low-pass filter with a cutoff frequency of $10 \mathrm{MHz}$ (3945 multichannel filter,

Krohn-Hite, Brockton, MA, USA). The filter bandwidth was chosen to capture 
all of the energy emitted from the transducer. The signal was displayed and averaged (100 averages) on an oscilloscope (9304A, Teledyne LeCroy, Chestnut Ridge, USA) and logged using an in-house LabVIEW program.

(a)

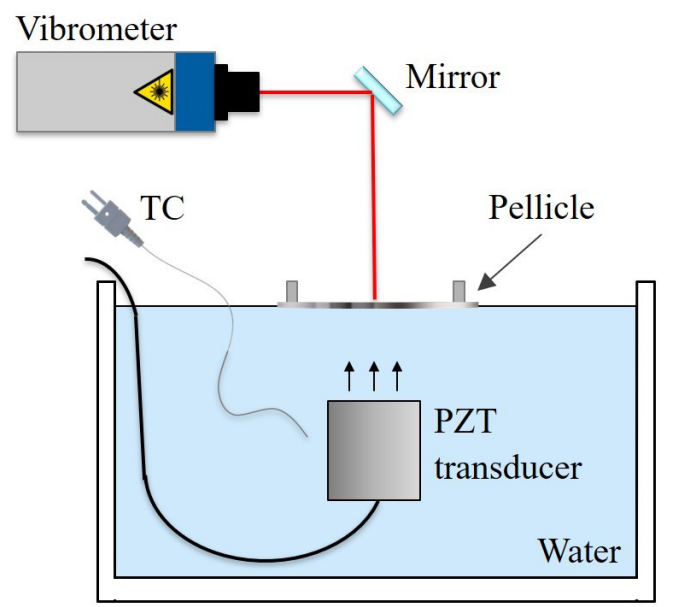

(b)

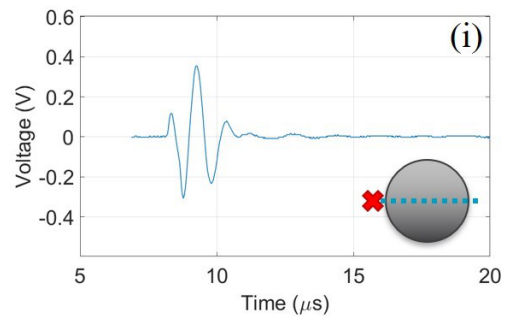

(ii)

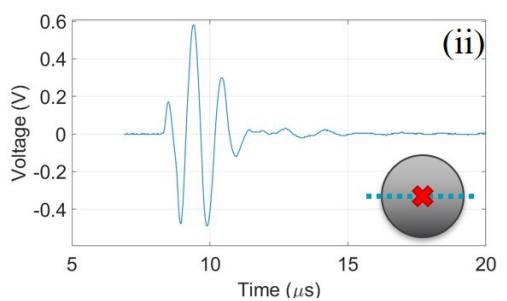

Figure 3: Laser vibrometry setup. (a) Transducer is positioned in a water tank with temperature control and J-type thermocouple (TC); thin reflective aluminium pellicle placed on water surface; laser vibrometer positioned on 2-axis motorised stage, with its beam directed perpendicular to pellicle using mirror. (b) Examples of displacement signal readouts for two scan positions (scan line: blue dashed line, scan position: red cross).

During the measurements, line scans were taken across the centre of the transducer face, with 41 points in $1 \mathrm{~mm}$ increments. Examples of voltage signal readouts for two scan positions are shown in Fig. 3(b). It can be seen that the pellicle displacement just outside the projection of the edge of the transducer case onto the pellicle (i) has a lower amplitude than the displacement induced at the centre of the transducer face (ii). These line scans were later combined into 2D plots using MATLAB.

Scans were made at temperatures from $46{ }^{\circ} \mathrm{C}$ to $22{ }^{\circ} \mathrm{C}$ in steps of $2{ }^{\circ} \mathrm{C}$ and repeated using different transducer drive settings in order to measure the relative displacement of the pellicle and assess how the relative pressure gener- 
ated by the transducer changes with temperature. The scans were made once

Figure 4 shows 2D plots of the pellicle displacement recorded for the three types of transducer excitations for the $1 \mathrm{MHz}$ transducer (top row) and $2.25 \mathrm{MHz}$ transducer (bottom row). For the PW and sine pulse excitations, it can be seen 

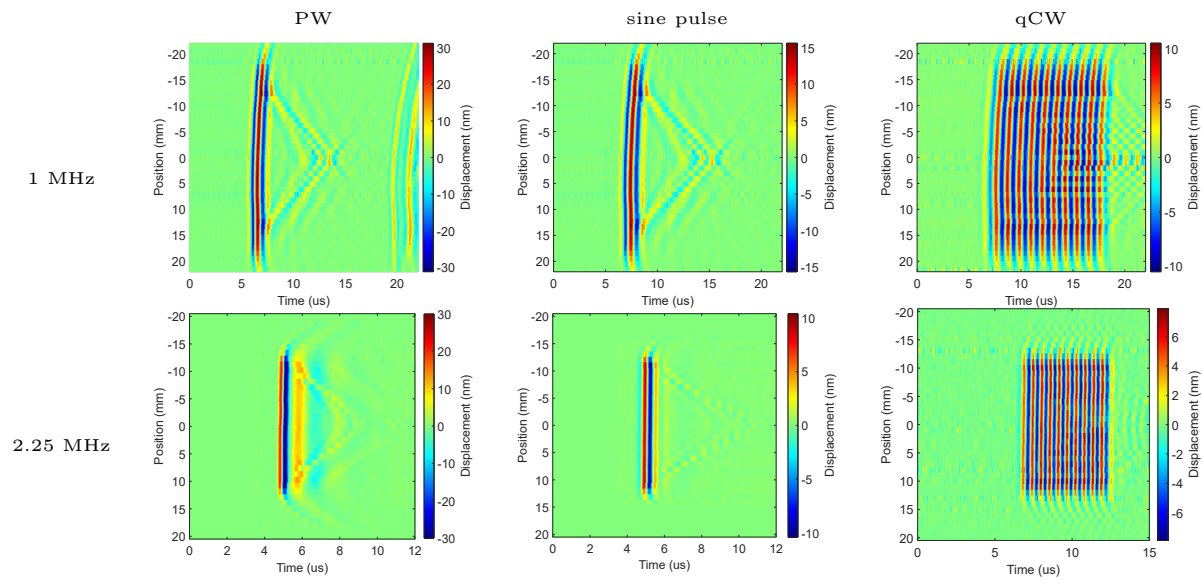

Figure 4: 2D plots showing the pellicle displacement for $1 \mathrm{MHz}$ and $2.25 \mathrm{MHz}$ transducers and three excitations: pulsed wave (PW), single-cycle (sine pulse) and long burst sinusoidal wave $(\mathrm{qCW})$, respectively.

that the ultrasound wave has two parts, namely a direct wave from the face of the transducer arriving at time $t=z / c_{w}$, followed by edge waves arriving at a subsequent time $t=\sqrt{a^{2}+z^{2}} / c_{w}$, where $a$ is the transducer radius and $z$ is the distance of a point on the transducer axis from the transducer, here corresponding to the transducer-pellicle separation. These edge waves are caused by the presence of laterally propagating plate waves that originate from the rim of the transducer and radiate into the surrounding fluid 9. Here, the edge and direct waves are clearly separated in time so that no interference occurs, and the edge waves were gated out in the data analysis. In the $\mathrm{qCW}$ case, on the other hand, the calculation suggests the edge waves arrive at $t=12 \mu \mathrm{s}$, thus overlapping with the direct wave. This can be seen in the 2D plot presented for the $1 \mathrm{MHz}$ transducer. However, as the edge waves have comparatively low amplitude these were not considered to affect the result.

In order to extract the relative change in pressure generated by the transducer as a function of temperature, the data was processed in the following manner. For long burst ( $\mathrm{qCW}$ ) excitation i.e. a narrowband signal with frequency content centered around the transducer's centre frequency, a windowed 
and zero-padded FFT was applied to the displacement signals spatially confined to the $10 \mathrm{~mm}$ centre region of the transducer. The amplitude information for each time series was extracted by taking the amplitude of the Fourier spectrum and the transducer centre frequency (using extractAmpPhase function in $\mathrm{k}$-Wave [36]). The mean amplitude of the 11 measurement points and their standard deviation at each measurement temperature were then used to calculate a relative change in pressure generated by the transducer between $22{ }^{\circ} \mathrm{C}$ to $46{ }^{\circ} \mathrm{C}$, as defined in Eq. 9. Here, a minimal change in beam shape is assumed, e.g., due to changes in the relative arrival time of the edge waves which depends on the speed of sound in water that changes by $3 \%$ from $22{ }^{\circ} \mathrm{C}$ to $46{ }^{\circ} \mathrm{C}$.

In the pulsed wave and single-cycle sinusoidal wave excitation measurements, the frequency content of the signals reflects the transducers' broadband response. In order to encompass all the information carried by the signals, the time derivative of the data was calculated using a second-order accurate central difference scheme (using gradientFD function in k-Wave 36]). The peak particle velocities were obtained as the maximum absolute value from the 10 line scans corresponding to the $10 \mathrm{~mm}$ centre region of the transducer. The mean and standard deviation were used in the calculation of the acoustic pressure generated by an ultrasound transducer as defined in Eq. 7.

\subsection{Electrical measurements}

Transducer electrical impedance measurements were performed using an impedance analyser (4294A, Agilent Technologies, Santa Clara, CA, USA). These measurements provided the electrical power values required to investigate how the radiated power from the transducer would change if the input electrical power was constant with temperature. This is reported as the temperaturedependent change in transducer efficiency $\epsilon$, defined as the ratio of the radiated acoustic power to electrical power. The electrical impedance measurements also enabled the assessment of how the pressure radiated from the transducer is altered by changes to the electrical signal supplied to it from a source of specific impedance. The reflection coefficients and the relevant energy transmission 
coefficients were calculated using the measured impedance data, as described below.

During the measurements, the transducers were immersed in a water bath lined with absorbers, the temperature of which was controlled using a thermostat (ECO RE415S Silver thermostat, Lauda GmbH, Lauda-Königshofen, Germany). The impedance analyser was calibrated using the Short-OpenLoad (SOL) technique [37] and measurements taken for a frequency range from $0.5 \mathrm{MHz}$ to $5 \mathrm{MHz}$ at temperatures between $22{ }^{\circ} \mathrm{C}$ and $46{ }^{\circ} \mathrm{C}$.

Electrical power $P$ to the transducer was calculated as:

$$
P=\frac{V_{R M S}^{2}}{|Z|} \cos (\arg (Z))
$$

where $V_{R M S}$ is the RMS voltage which was kept constant at $10 \mathrm{~V}$ (corresponding to a peak positive voltage of $14 \mathrm{~V}),|Z|$ is the modulus of the measured complex impedance and $\arg (Z)$ was calculated as a 2 -argument inverse tangent of the real and complex part of the measured impedance [38.

In order to assess how the electrical drive system could affect the temperaturedependent transducer output, reflection coefficients $R$ were calculated assuming an interface between the transducer $\left(Z_{T}\right)$ and a source with a fixed characteristic impedance $\left(Z_{C}\right)$ :

$$
R=\left|\frac{Z_{C}-Z_{T}}{Z_{C}+Z_{T}}\right| .
$$

The coefficients were calculated for temperatures between $22{ }^{\circ} \mathrm{C}$ and $46{ }^{\circ} \mathrm{C}$, using the measured transducer impedance values $Z_{T}$ at the corresponding temperature. Two cases were investigated, with fixed characteristic impedances $Z_{C}$ of $50+0 \mathrm{j} \Omega$ and a complex impedance of $2-11 \mathrm{j}$. The $50 \Omega$ case was considered for scenarios when using a drive system where function generator and amplifiers have input and output impedances of $50 \Omega$, and where all interconnecting cables behave as $50 \Omega$ transmission lines. In contrast, the output impedance of a pulser is likely to be a low real impedance plus capacitance but will not be constant. This variation is difficult to quantify as it arises from the avalanche discharge of a capacitor bank in the output stage of the pulser, which may vary on a 
nanosecond timescale. The complex impedance $2-11 \mathrm{j}$ is chosen as a representative value. Energy transmission coefficients were calculated as $T_{e}=1-R_{e}$, where $R_{e}=R^{2}$.

\subsection{Uncertainty evaluation}

Measurement uncertainty was evaluated following the guide to the expression of uncertainty in measurement [39]. The expanded measurement uncertainty quoted in the results section was determined using both Type A (random) and 325 Type B (systematic) uncertainty evaluations and is given as the standard uncertainty multiplied by a coverage factor, $\mathrm{k}=2$, providing a coverage probability of approximately $95 \%(\mathrm{p}=0.95)$, according to the method recommended in 39, 40. Type B uncertainties arise from several sources, which were independently evaluated or quoted from the available literature, as briefly described in

330 Table A.1 in Appendix A. The combined standard uncertainties of quantities derived from the measured data were obtained according to the expression of propagation of uncertainty in measurement for uncorrelated input quantities [40].

An uncertainty budget for determination of ultrasound power output using radiation force balances was calculated according to IEC 61161 [20]. Type A uncertainty for each target type and measurement temperature was assessed from the four on-off transition differences. Type B uncertainties arise from several sources which were independently evaluated at different frequencies. Some of these include the linearity and resolution of the balance system, target im340 perfections, setup misalignment, acoustic streaming, environmental influences, temperature variations, oscilloscope resolution and the linearity of the amplifier [20, 39, 41].

For the laser vibrometry measurements, uncertainty budget calculations were performed as described in 33. Type A uncertainty for each transducer drive setting and measurement temperature was assessed from the pellicle displacement amplitudes of scan points across the transducer face. Sources of type B uncertainties that were taken into account included pellicle properties, vibrom- 
eter noise, decoder linearity, electrical load correction, distance dependence and repeatability of the acoustic field, oscilloscope resolution and the linearity of the amplifier [33, 39.

Sources of uncertainty considered for the electrical measurements included the errors in real and imaginary parts of the measured complex impedance, VNA uncertainty, and water and transducer temperature.

\subsection{Statistical analysis}

In order to confirm the significance of the observed trends in the results obtained from both methods, weighted least-squares (WLS) regression was used [42. Here, an additional scale factor (i.e. weight) was included in the fitting process, where the weight is the inverse of the square of the variance of the data point.

\section{Results and discussion}

\subsection{Continuous wave measurements}

\subsubsection{Comparison of RFB targets}

First, a comparison between the two radiation force balance configurations utilising an absorbing and reflecting target was performed as shown in Fig. 5 These measurements were done using the $2.25 \mathrm{MHz}$ PZT transducer. It can be seen that the results for both RFB targets are in good agreement and exhibit an increasing trend in the transducer radiated power with temperature from $22{ }^{\circ} \mathrm{C}$ to $46{ }^{\circ} \mathrm{C}$, corresponding to approximately $1.4 \%$ increase per degree Celsius. The expanded measurement uncertainty in the radiated power for the absorbing target was around $5 \%$ over the whole temperature range, while for the reflecting target it varied between $4 \%$ and $8 \%$. These were mostly due to temperature variations and drifts in baseline levels and readout levels of the balance caused by the rapid evaporation rate of water which meant the weight experienced by the balance was continuously decreasing. The results obtained in 
from increased measurement errors as previously described in Section 2.1.2. Due to various advantages of using this configuration, all subsequent radiation force balance measurements utilised the absorbing target only.

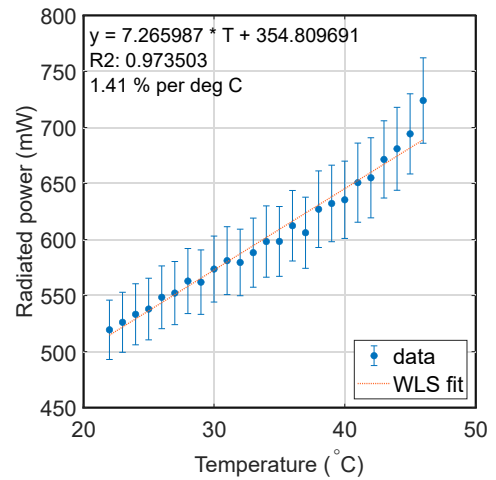

(a)

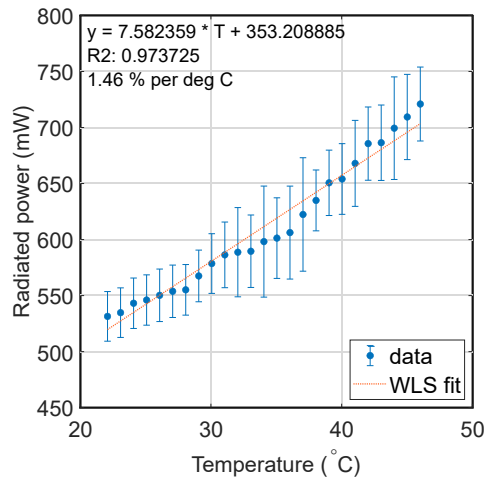

(b)

Figure 5: Power radiated from the $2.25 \mathrm{MHz}$ transducer at temperatures between $22{ }^{\circ} \mathrm{C}$ and $46{ }^{\circ} \mathrm{C}$ as determined using the radiation force balance configurations with (a) absorbing target and (b) reflecting target. Error bars represent the expanded uncertainty $(\mathrm{p}=0.95)$. The dotted lines represent the weighted least-squares (WLS) regression fit.

\subsubsection{Comparison of RFB and laser vibrometry results}

Figure 6 shows the temperature dependence of the relative pressure radiated from the $1 \mathrm{MHz}$ and $2.25 \mathrm{MHz}$ transducers driven with a continuous sinusoidal wave (or $\mathrm{qCW}$ ), as determined from the radiation force balance measurements utilising the absorbing target and laser vibrometry. The measured quantities, namely radiated power in RFB and pellicle displacement in laser vibrometry, were transformed to radiated pressure using equations 6 and 9, respectively, in order to allow the intercomparison between the two techniques. The values for the temperature-dependent density and speed of sound of water were obtained from Jones [25] and Marczak [26, respectively. The results are presented for temperatures between $22{ }^{\circ} \mathrm{C}$ to $46{ }^{\circ} \mathrm{C}$, and are normalised to the values at 22 degrees Celsius, and trends described with a linear equation $\left(\mathrm{R}^{2}>0.9\right)$.

The results from RFB and laser vibrometry measurements for both transducers are in good agreement and exhibit an increasing trend in the transducer 


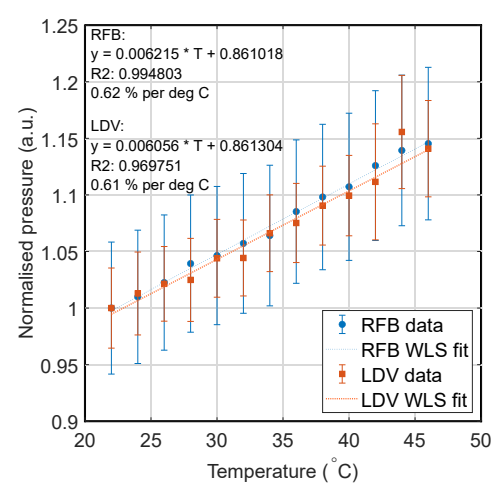

(a)

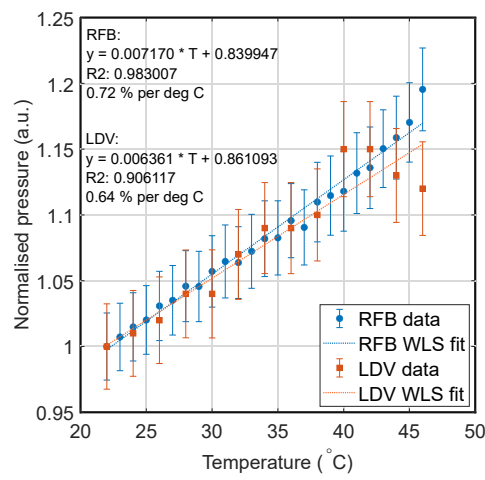

(b)

Figure 6: Temperature-dependent pressure radiated from the (a) $1 \mathrm{MHz}$ and (b) $2.25 \mathrm{MHz}$ transducer as determined from the radiation force balance measurements (blue circles) and laser vibrometry (orange squares). The results are presented for temperatures between $22^{\circ} \mathrm{C}$ and $46{ }^{\circ} \mathrm{C}$, normalised to $22^{\circ} \mathrm{C}$. Error bars represent the expanded uncertainty $(\mathrm{p}=0.95)$. The dotted lines represent the weighted least-squares (WLS) regression fit.

output with temperature when driven at constant voltage. Both transducers show consistent variation with temperature, corresponding to approximately $14 \%$ increase between $22{ }^{\circ} \mathrm{C}$ and $46{ }^{\circ} \mathrm{C}$ ( or $0.6 \%$ per ${ }^{\circ} \mathrm{C}$ ).

The expanded measurement uncertainty in the radiated acoustic power for the $1 \mathrm{MHz}$ transducer (Fig. 6(a)) was around $12 \%$ over the whole temperature range, while the combined standard uncertainty of the pressure is $6 \%$. For this measurement the Aptflex F28P absorbing target was used. This has a lower limit for the suitability of application of $1 \mathrm{MHz}$ thus explaining the larger uncertainties observed. The combined standard uncertainty of the pressure calculated from radiation force balance measurements using the HAM A-LF target for the $2.25 \mathrm{MHz}$ transducer (Fig. 6(b)) is around $2.5 \%$. As for the results obtained using laser vibrometry, the expanded measurement uncertainty in the measured displacement and the combined standard uncertainty of the calculated pressure was around $3 \%$ across the entire temperature range for both transducers. 


\subsubsection{Transducer efficiency}

Using the impedance analyser, the complex impedances of the two PZT

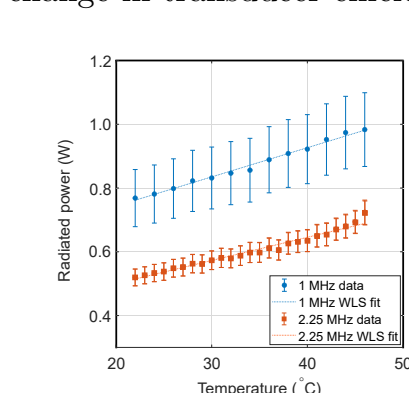

(a)

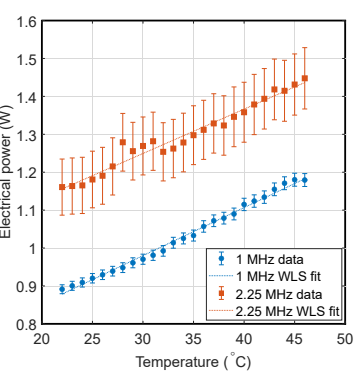

(b)

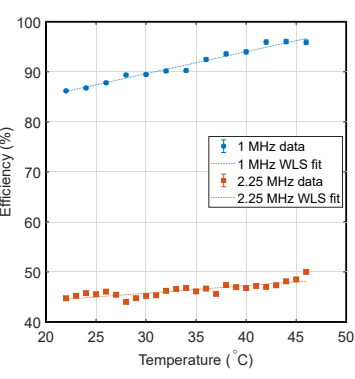

(c)

Figure 7: Temperature-dependent (a) radiated power with constant drive voltage, (b) electrical power to the transducer, (c) efficiency of the $1 \mathrm{MHz}$ (blue circles) and $2.25 \mathrm{MHz}$ (orange squares) PZT transducer between $22^{\circ} \mathrm{C}$ and $46{ }^{\circ} \mathrm{C}$. Error bars represent the expanded uncertainty $(\mathrm{p}=0.95)$. The dotted lines represent the weighted least-squares (WLS) regression fit.

The measured resistance of the $1 \mathrm{MHz}$ transducer decreased by $30 \%$, while its reactive impedance increased by $22 \%$ within the measurement range. The $2.25 \mathrm{MHz}$ transducer's resistance and reactive impedance increased by $9 \%$ and $7 \%$, respectively. When keeping the input peak positive voltage constant at $14 \mathrm{~V}$, the radiated power (Fig. 7(a)) from the $1 \mathrm{MHz}$ transducer (shown in blue) increased by $28 \%$, while for the $2.25 \mathrm{MHz}$ transducer (shown in orange) it increased by $40 \%$. The corresponding electrical power inputs to the transducer (Fig. $7(\mathrm{~b}))$ increased by $32 \%(1 \mathrm{MHz})$ and $25 \%(2.25 \mathrm{MHz})$. Scaling the ${ }_{425}$ radiated power with the temperature-dependent electrical power input to the 
transducer provides the transducer efficiency (Fig. 7( (c)), here used as a measure of how the acoustic power radiated from the transducer would change if the input electrical power was constant with temperature. The efficiency of the $1 \mathrm{MHz}$ transducer increased from $86 \%$ at $22{ }^{\circ} \mathrm{C}$ to $96 \%$ at $46{ }^{\circ} \mathrm{C}$, while for the $2.25 \mathrm{MHz}$ transducer it increased from $45 \%$ to $50 \%$. Across both transducers, this corresponds to a relative increase in efficiency of approximately $0.5 \%$ per ${ }^{\circ} \mathrm{C}$.

The expanded measurement uncertainties in the measured resistance and reactive impedance were both approximately $1 \%$ for the $1 \mathrm{MHz}$ transducer, while for the $2.25 \mathrm{MHz}$ transducer these were around $5 \%$ and $1 \%$, respectively. The expanded measurement uncertainty in the radiated power and the electrical power input to the transducer were $12 \%$ and $2 \%$ for the $1 \mathrm{MHz}$, while for the $2.25 \mathrm{MHz}$ transducer these were around $5 \%$ and $6 \%$ over the whole temperature range, respectively. The propagated uncertainty of the transducer efficiency was around $14 \%$ for the $1 \mathrm{MHz}$ transducer and $11 \%$ for the $2.25 \mathrm{MHz}$ transducer. Error bars in Fig. 7(c) are omitted for clarity.

\subsection{Investigation of excitation source types using laser vibrometry}

The effect of excitation source type on the temperature dependency of transducer output was investigated using laser vibrometry. The temperature-dependent plots for the measured displacements, as well as calculated particle velocities and pressures can be found in Appendix B. Figure 8 shows the generated pressure radiated from the transducers obtained for temperatures between $22{ }^{\circ} \mathrm{C}$ to $46{ }^{\circ} \mathrm{C}$, calculated using Eq. 7 for broadband pulses and Eq. 9 for narrowband pulses. All results are normalised to values at 22 degrees Celsius, and trends described

450 with a linear fit.

The results for the $1 \mathrm{MHz}$ transducer exhibit similar increasing trends for all excitation types, corresponding to an average increase of $15 \%$ from $22{ }^{\circ} \mathrm{C}$ to $46{ }^{\circ} \mathrm{C}$. The uncertainty in the measured displacement, and the propagated uncertainty of the calculated pressure presented in Fig. 8 for the $1 \mathrm{MHz}$ transducer was around $3 \%$ for PW and $\mathrm{qCW}$ case, and $4 \%$ for single-cycle excitation, and 
$1 \mathrm{MHz}$
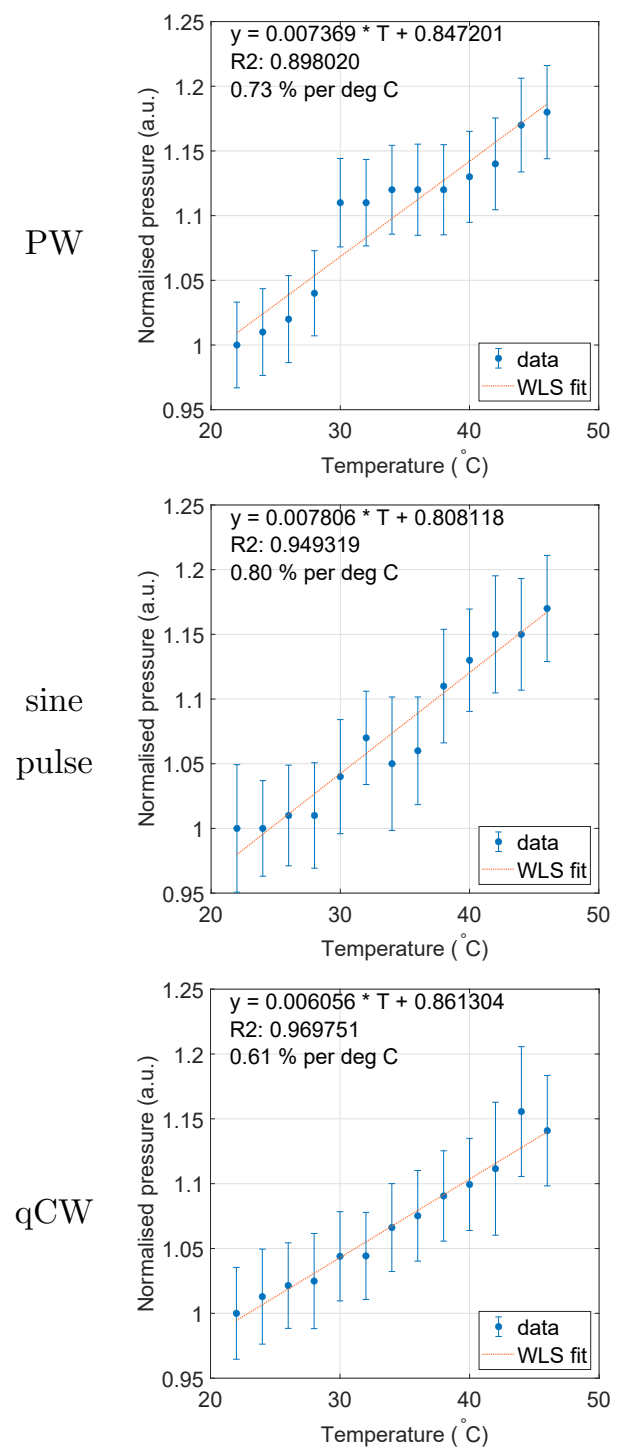

$2.25 \mathrm{MHz}$
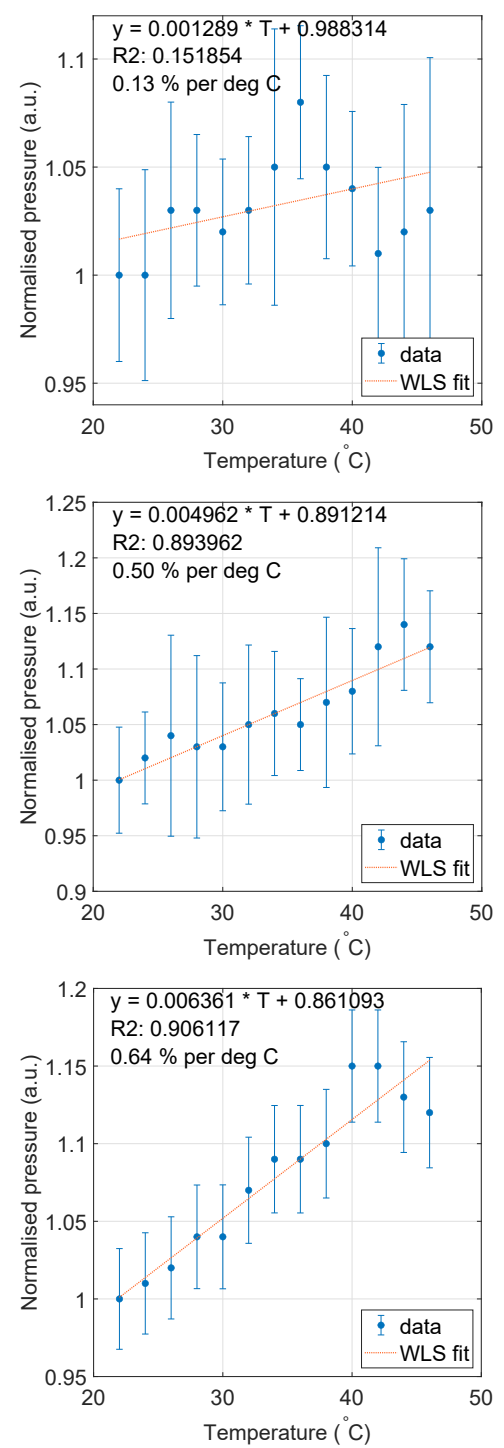

Figure 8: Relative change in pressure generated from two PZT transducers excited by a pulsed wave (PW), single-cycle (sine pulse) and long burst (qCW) sinusoidal wave excitation. The results are presented for temperatures between $22{ }^{\circ} \mathrm{C}$ and $46{ }^{\circ} \mathrm{C}$, normalised to $22{ }^{\circ} \mathrm{C}$. Error bars represent the expanded uncertainty $(\mathrm{p}=0.95)$. The dotted lines represent the weighted least-squares (WLS) regression fit. 
was consistent across the entire temperature range.

In the case of the $2.25 \mathrm{MHz}$ transducer, the results for narrowband excitation are similar to the $1 \mathrm{MHz}$ transducer and show an increase in generated pressure of approximately $12 \%$ over the temperature range from $22{ }^{\circ} \mathrm{C}$ to $46{ }^{\circ} \mathrm{C}$. from a source of specific impedance were investigated. The energy transmission coefficients were calculated for the interface between the PZT transducer and an excitation source with a fixed characteristic impedance of (i) $50+0 \mathrm{j}$, corresponding to a signal generator and amplifier, and a (ii) complex impedance of 485 On the other hand, when using a pulser there is no clear trend in the temperature dependence of the generated pressure radiated from the transducer. These measurements were repeated using different configurations including several pellicle types, measurements of water surface and direct transducer surface measurements through an anti-reflective glass window. All configurations displayed similar results, thus providing confidence in the measurement results. The uncertainty in the measured displacement, and the propagated uncertainty of the calculated pressure for the $2.25 \mathrm{MHz}$ transducer, varied between $3.5 \%$ and $7 \%$ for PW, and $4 \%$ to $8.5 \%$ for sine pulse excitation, while in qCW case it was consistent around $3 \%$ across the entire temperature range.

One possible explanation for the observed difference in results between the three transducer excitations for the $2.25 \mathrm{MHz}$ transducer was investigated by considering the electrical impedance properties of the two PZT transducers. Firstly, the resistive versus reactive impedance plots (Fig. 9) of the transducers were analysed. The plot for the $2.25 \mathrm{MHz}$ transducer exhibited a behaviour atypical from the IEEE standard 178 on piezoelectricity [43]. This standard implies transducers normally exhibit a resistive vs. reactive loop, as was observed for the $1 \mathrm{MHz}$ transducer (a) but not for the $2.25 \mathrm{MHz}$ transducer (b). This would indicate the latter to have a non-standard electrical impedance matching.

Secondly, the effect of temperature change on the electrical input to the transducer, its impedance and the changes to the electrical signal supplied to it $2-11 \mathrm{j}$, representative of a pulser. The results for the $2.25 \mathrm{MHz}$ transducer are presented in Fig. 10 for temperatures between $22^{\circ} \mathrm{C}$ and $46{ }^{\circ} \mathrm{C}$. 


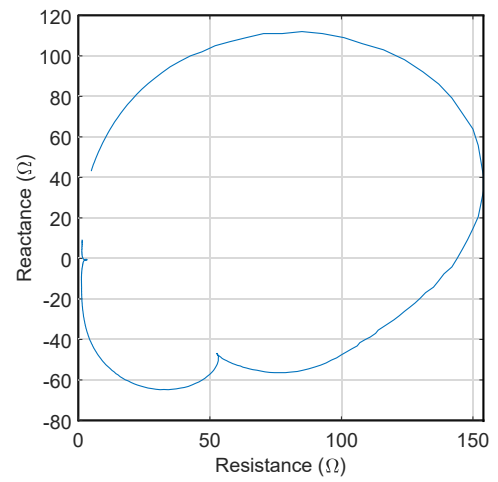

(a)

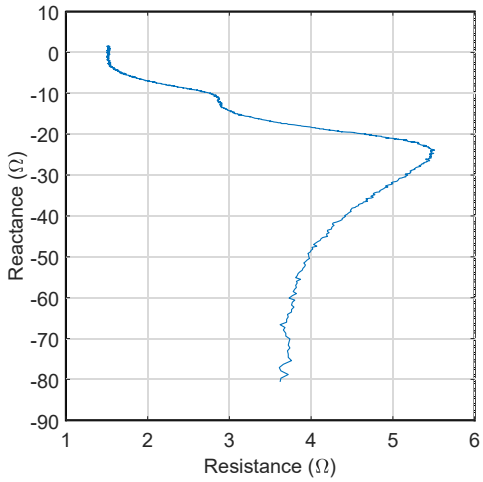

(b)

Figure 9: Resistive versus reactive impedance plots for the (a) $1 \mathrm{MHz}$ and (b) $2.25 \mathrm{MHz} \mathrm{PZT}$ transducers.

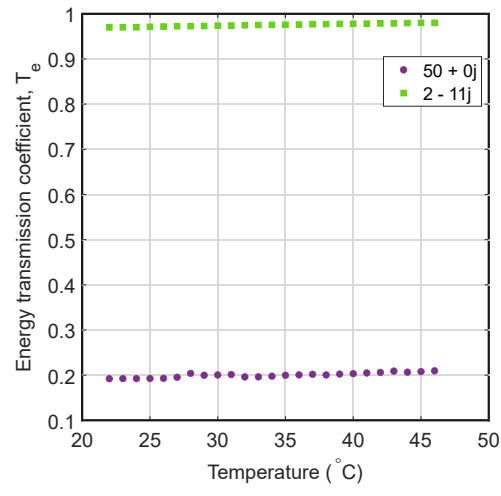

(a)

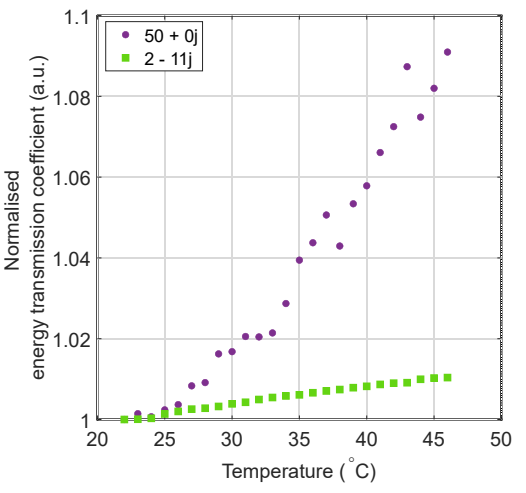

(b)

Figure 10: Energy transmission coefficient $\left(T_{e}\right)$ between the $2.25 \mathrm{MHz}$ PZT transducer and an excitation source with a fixed characteristic impedance of $50+0 \mathrm{j}$ (purple circles), and complex impedance of $2-11 \mathrm{j}$ (green squares). Results presented for temperatures between $22^{\circ} \mathrm{C}$ and $46^{\circ} \mathrm{C}$, as (a) absolute values and (b) relative change. 
At the interface between the $2.25 \mathrm{MHz}$ transducer and a source impedance of $50+0 \mathrm{j}$, the energy transmission coefficient increases with temperature by $12 \%$ within the measurement range from $22{ }^{\circ} \mathrm{C}$ to $46{ }^{\circ} \mathrm{C}$. This indicates more energy is arriving at the transducer at higher temperatures, which is an effect observed in the laser vibrometry result for the transducer driven with a signal generator and amplifier (sine pulse and $\mathrm{qCW}$ case). In the case of a complex source impedance, however, there is very little relative change $(1 \%)$ in the energy transmission coefficient as a function of temperature and thus no changes in the transducer output are observed in the laser vibrometry measurements. This hypothesis is supported by a similar analysis for the $1 \mathrm{MHz}$ transducer, where the energy transmission coefficient was calculated to significantly increase for both driving conditions. It should be noted, however, that transducer output is dependent on numerous different variables not investigated in this study and these finding should thus not be considered absolute.

\section{Summary and Discussion}

The temperature-dependent output of two single-element unfocused PZT transducers (with centre frequencies $1 \mathrm{MHz}$ and a $2.25 \mathrm{MHz}$ ) immersed in water between $22{ }^{\circ} \mathrm{C}$ to $46{ }^{\circ} \mathrm{C}$ was investigated. When driven using a continuous wave $(\mathrm{CW})$ or quasi-CW excitation with constant input voltage using a signal generator and amplifier matched to $50 \Omega$, the pressure radiated from the two transducers increased by approximately $0.6 \%$ per ${ }^{\circ} \mathrm{C}$ over the measurement range. This was primarily due to an increase in transducer efficiency, which exhibited a relative increase of approximately $0.5 \%$ per ${ }^{\circ} \mathrm{C}$.

Two independent measurement methods were used for the study, namely, radiation force balance (RFB) and laser vibrometry. For the RFB measurements using CW excitation, close agreement was observed using both absorbing and reflecting targets. Additionally, close agreement was observed between RFB measurements using an absorbing target and CW excitation, and laser vibrom- 
Additional measurements using laser vibrometry and a single-cycle sine pulse excitation with a matched signal chain showed similar trends to the quasi-CW case. However, when driven with a pulser (which is not electrically matched), the two transducers exhibited different behaviour depending on their electrical impedance. For the $1 \mathrm{MHz}$ transducer, the output using the pulsed excitation was similar to the $\mathrm{CW}$ case. However, for the $2.25 \mathrm{MHz}$ transducer, the output did not show a strong temperature dependence. These results can largely be explained by differences in the electrical impedance of the $2.25 \mathrm{MHz}$ transducer (which is closer to the impedance of the pulser), which results in a negligible relative change in energy transmission coefficient with temperature.

In general, the output of PZT transducers will depend on temperature. It is thus recommended that for applications where PZT transducers are used at temperatures significantly different from the characterisation temperature, a measurement of the transducer output is performed using one of the described methods and correction for this effect taken into account. Moreover, the results for the pulsed case suggest the measurement of the temperature-dependent transducer output must be done using the electrical drive system relevant to the target application. Note, air-backed transducers (e.g., as commonly used in ultrasound therapy) and transducers without an acoustic matching layer were not investigated in this study, although similar trends might be expected given the temperature-dependent changes in the properties of PZT observed in previous studies as discussed in Sec. 1.2.

Regarding the two measurement approaches used in the study, they each have advantages and disadvantages. Radiation force balances are affordable ${ }_{40}$ and more readily available in ultrasound measurement laboratories. They are straightforward to setup and use, however, this approach may not be appropriate for all transducers. RFBs have relatively low sensitivities and can only detect powers above 10-20 $\mathrm{mW}(20]$, 44], 45]). Consequently, not all excitation regimes can be used during these measurements. Additionally, RFBs can

545 only measure the total output power of a transducer (no spatial information is given on the produced field). Laser vibrometry on the other hand requires more 
expensive and specialised equipment, as well as a complex setup. However, it can be used for all excitation regimes, and also captures the time domain displacement, allowing spatial information about the acoustic field (including edge waves) to be captured. The frequency range for the RFB is limited by the design of the acoustic target, while the frequency range for the laser vibrometry is limited by the digitiser and decoder. In practice, both techniques cover the frequency range of interest to medical ultrasonics. Both methods as implemented for this study are limited to measuring unfocused transducers driven at moderate acoustic power levels under which the wave propagation is linear. However, methods to extend RFB measurements to much higher power levels and highly focused fields have previously been described [46, 47, and measuring the temperature-dependent output of such transducers would make for interesting future work.

\section{Declarations}

\subsection{Competing interests}

The authors declare that they have no competing interests.

\subsection{Funding}

This work was supported by the Engineering and Physical Sciences Research Council, UK.

\subsection{Author's contributions}

MB: Formal analysis, Investigation, Methodology, Validation, Visualization, Writing - original draft; PF: Methodology, Writing - review \& editing; AH: Formal analysis, Writing - review \& editing; SR: Methodology, Writing - review \& editing; BZ: Conceptualization, Funding acquisition, Supervision; BC: Supervision, Writing - review \& editing; PM: Formal analysis, Methodology, Supervision, Writing - review \& editing; BT: Funding acquisition, Investigation, Supervision, Writing - review \& editing. 


\subsection{Acknowledgements}

575 The authors would like to thank Nicole Anstey for help with the radiation force balance measurements at NPL, and Natasha Sheard for the initial laser vibrometry measurements. A special thanks to the Ultrasound and Underwater Acoustics team at NPL for useful discussions. 
${ }_{580}$ Appendix A.

Table A.1: Uncertainty sources and their contributions, expressed as a percentage (\%).

\begin{tabular}{|c|c|c|}
\hline Source of uncertainty & $\begin{array}{l}\text { Standard uncertainty }(\mathrm{k}=1) \text {, } \\
\text { values in } \%\end{array}$ & Description \\
\hline \multicolumn{3}{|l|}{ Radiation force balance } \\
\hline Type A & 5.00 & Random/repetitions \\
\hline $\begin{array}{l}\text { Balance system including target sus- } \\
\text { pension }\end{array}$ & 0.07 & Measured in-house \\
\hline Linearity of the balance & 0.06 & Measured in-house \\
\hline Resolution of the balance & 0.24 & From balance specifications \\
\hline $\begin{array}{l}\text { Extrapolation to the moment of } \\
\text { switching the ultrasonic transducer }\end{array}$ & 0.12 & Measured in-house \\
\hline Target imperfections & 0.70 & Measured in-house \\
\hline Transducer misalignment & 0.14 & Measured in-house \\
\hline Water temperature & 0.12 & Calculated from 26 \\
\hline Finite target size & 0.44 & Measured in-house \\
\hline Plane-wave assumption & 0.17 & Measured in-house \\
\hline Environmental influences & 0.30 & Measured in-house \\
\hline Transducer temperature & 0.14 & Measured in-house \\
\hline Target type & 1.15 & Measured in-house \\
\hline $\begin{array}{l}\text { Difference between turn-on and turn- } \\
\text { off value }\end{array}$ & 0.76 & Measured in-house \\
\hline Excitation voltage measurement & 0.35 & Measured in-house \\
\hline \multicolumn{3}{|l|}{ Laser vibrometry } \\
\hline Type A & $\begin{array}{l}\text { Between } 3.00 \% \text { and } 8.50 \% \text {, depending } \\
\text { on the transducer excitation mode }\end{array}$ & Random/repetitions \\
\hline Vibrometer noise & 0.02 & Interpolated from 33 \\
\hline Photodiode of vibrometer & 0.27 & Interpolated from 33 \\
\hline Electrical load correction & 0.58 & Interpolated from 33 \\
\hline Repeatability of acoustic field & 0.15 & Measured in-house \\
\hline Distance dependence of the field & 0.15 & Interpolated from 33 \\
\hline Transmission factor of pellicle & 0.07 & Interpolated from 33 \\
\hline Decoder linearity & $1 \%$ for $\mathrm{qCW}, 5 \%$ for $\mathrm{PW}$ & From decoder specifications \\
\hline Oscilloscope resolution & 0.10 & Table 1 in 48 \\
\hline Oscilloscope linearity and distortion & 0.29 & From oscilloscope specifications \\
\hline $\begin{array}{l}\text { Oscilloscope temperature and time de- } \\
\text { pendance }\end{array}$ & 0.03 & Table 1 in 48 \\
\hline Alignment and time delay & 0.10 & Measured in-house \\
\hline Transducer misalignment & 0.14 & Measured in-house \\
\hline Water temperature & 0.12 & Calculated from 26 \\
\hline Finite target size & 0.44 & Measured in-house \\
\hline Plane-wave assumption & 0.17 & Measured in-house \\
\hline Environmental influences & 0.25 & Measured in-house \\
\hline \multicolumn{3}{|l|}{ Electrical impedance } \\
\hline Type A for R & 5.00 & Random \\
\hline Type A for $\mathrm{X}$ & 1.00 & Random \\
\hline Transducer temperature & 0.14 & Measured in-house \\
\hline Water temperature & 0.12 & Calculated from 26 \\
\hline
\end{tabular}




\section{Appendix B.}

Figures $\mathrm{B} .1$ and $\mathrm{B} .2$ show the measured displacements, calculated particle velocities and generated pressures radiated from the $1 \mathrm{MHz}$ and $2.25 \mathrm{MHz}$ transducer, respectively.
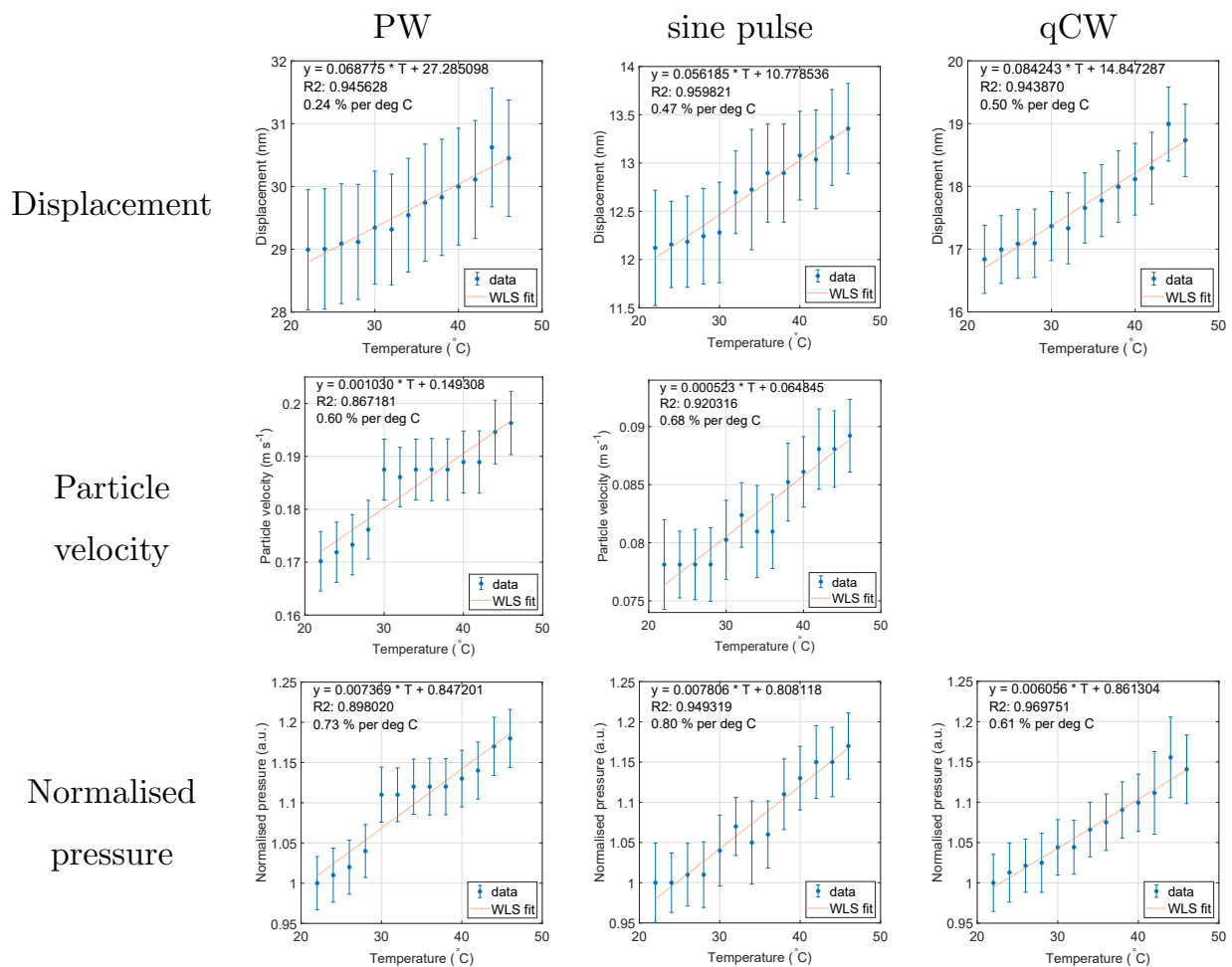

Figure B.1: $1 \mathrm{MHz}$ transducer. Temperature-dependent change in displacements measured using laser vibrometry as well as calculated particle velocities and generated pressures. 

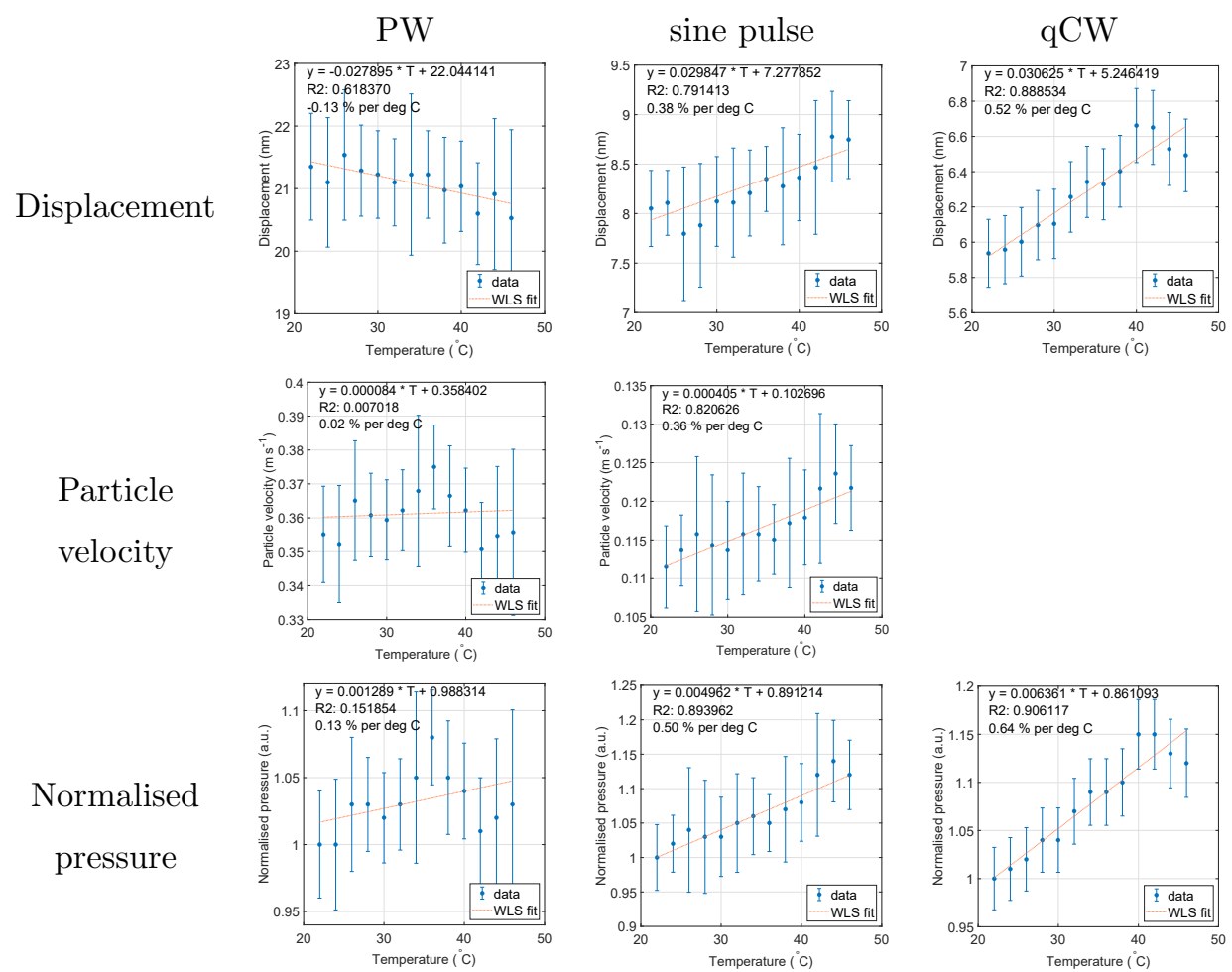

Figure B.2: $2.25 \mathrm{MHz}$ transducer. Temperature-dependent change in displacements measured using laser vibrometry as well as calculated particle velocities and generated pressures. 


\section{References}

[1] W. Legon, L. Ai, P. Bansal, J. K. Mueller, Neuromodulation with singleelement transcranial focused ultrasound in human thalamus, Human Brain Mapping 39 (5) (2018) 1995-2006.

[2] E. Martin, B. Treeby, Investigation of the repeatability and reproducibility of hydrophone measurements of medical ultrasound fields, The Journal of the Acoustical Society of America 145 (3) (2019) 1270-1282.

[3] O. A. Sapozhnikov, S. A. Tsysar, V. A. Khokhlova, W. Kreider, Acoustic holography as a metrological tool for characterizing medical ultrasound sources and fields, The Journal of the Acoustical Society of America 138 (3) (2015) 1515-1532.

[4] International Electrotechnical Commission, IEC 62127-2: Ultrasonics - Hydrophones, Part 2: Calibration for ultrasonic fields up to $40 \mathrm{MHz}$ (2013).

[5] International Electrotechnical Commission, IEC 62127-1: Ultrasonics - Hydrophones, Part 1: Measurement and characterization of medical ultrasonic fields up to $40 \mathrm{MHz}$ (2013).

[6] R. Preston, S. Robinson, B. Zeqiri, T. Esward, P. Gélat, N. Lee, Primary calibration of membrane hydrophones in the frequency range $0.5 \mathrm{MHz}$ to $60 \mathrm{MHz}$, Metrologia 36 (4) (1999) 331.

[7] International Electrotechnical Commission, IEC 61689: Ultrasonics. Physiotherapy systems. Field specifications and methods of measurement in the frequency range $0,5 \mathrm{MHz}$ to $5 \mathrm{MHz}$ (2013).

[8] B. Sun, A. De Salles, Neurosurgical treatments for psychiatric disorders, Springer, 2014, Ch. 12.

[9] R. S. Cobbold, Foundations of biomedical ultrasound, Oxford university press, 2006. 
[10] J. Gallego-Juarez, Piezoelectric ceramics and ultrasonic transducers, Journal of Physics: Scientific Instruments 22 (10) (1989) 804.

[11] D. Wang, Y. Fotinich, G. P. Carman, Influence of temperature on the electromechanical and fatigue behavior of piezoelectric ceramics, Journal of Applied Physics 83 (10) (1998) 5342-5350.

[12] Q. Zhang, H. Wang, N. Kim, L. Cross, Direct evaluation of domain-wall and intrinsic contributions to the dielectric and piezoelectric response and their temperature dependence on lead zirconate-titanate ceramics, Journal of Applied Physics 75 (1) (1994) 454-459.

[13] R. Wolf, S. Trolier-McKinstry, Temperature dependence of the piezoelectric response in lead zirconate titanate films, Journal of Applied Physics 95 (3) (2004) 1397-1406.

[14] N. Horchidan, C. Ciomaga, R. Frunza, C. Capiani, C. Galassi, L. Mitoseriu, A comparative study of hard/soft PZT-based ceramic composites, Ceramics International 42 (7) (2016) 9125-9132.

[15] Z. Zhuang, M. J. Haun, S.-J. Jang, L. E. Cross, Composition and temperature dependence of the dielectric, piezoelectric and elastic properties of pure PZT ceramics, IEEE transactions on ultrasonics, ferroelectrics, and frequency control 36 (4) (1989) 413-416.

[16] C. Miclea, C. Tanasoiu, L. Amarande, C. Miclea, C. Plavitu, M. Cioangher, L. Trupina, C. Miclea, C. David, Effect of temperature on the main piezoelectric parameters of a soft PZT ceramic, Rom. J. Inf. Sci. Technol 10 (3) (2007) 243-250.

[17] R. Georges Sabat, B. K. Mukherjee, W. Ren, G. Yang, Temperature dependence of the complete material coefficients matrix of soft and hard doped piezoelectric lead zirconate titanate ceramics, Journal of Applied Physics 101 (6) (2007) 064111. 
[18] S. Sherrit, G. Yang, H. Wiederick, B. Mukherjee, Temperature dependence of the dielectric, elastic and piezoelectric material constants of lead zirconate titanate ceramics, in: Proceedings of the International Conference on Smart Materials, Structures and Systems, 1999, pp. 7-10.

[19] F. Xu, S. Trolier-McKinstry, W. Ren, B. Xu, Z.-L. Xie, K. Hemker, Domain wall motion and its contribution to the dielectric and piezoelectric properties of lead zirconate titanate films, Journal of Applied Physics 89 (2) (2001) 1336-1348.

[20] International Electrotechnical Commission, IEC 61161: Ultrasonics - Power measurement - Radiation force balances and performance requirements (2013).

[21] K. Beissner, Radiation force and force balances, Ultrasonic Exposimetry (1993) 163-168.

[22] K. Beissner, Minimum target size in radiation force measurements, The Journal of the Acoustical Society of America 76 (5) (1984) 1505-1510.

[23] R. C. Preston, Output Measurements for Medical Ultrasound, Springer Science \& Business Media, 2012.

${ }_{655}^{6}$ [24] G. Torr, The acoustic radiation force, American Journal of Physics 52 (5) (1984) 402-408.

[25] F. E. Jones, G. L. Harris, ITS-90 density of water formulation for volumetric standards calibration, Journal of Research of the National Institute of Standards and Technology 97 (3) (1992) 335.

[26] W. Marczak, Water as a standard in the measurements of speed of sound in liquids, The Journal of the Acoustical Society of America 102 (5) (1997) $2776-2779$.

[27] B. Zeqiri, L. Wang, P. Miloro, S. P. Robinson, A radiation force balance target material for applications below $0.5 \mathrm{MHz}$, Ultrasound in Medicine \& Biology 46 (9) (2020) 2520-2529. 
[28] Y. Sutton, A. Shaw, B. Zeqiri, Measurement of ultrasonic power using an acoustically absorbing well, Ultrasound in Medicine \& Biology 29 (10) (2003) 1507-1513.

[29] A. Shaw, M. Hodnett, Calibration and measurement issues for therapeutic ultrasound, Ultrasonics 48 (4) (2008) 234-252.

[30] J. Haller, K. Jenderka, A. Shaw, G. Durando, B. Karaböce, Metrology of high-intensity therapeutic ultrasound within the EMRP project 'External Beam Cancer Therapy'. Characterization of sources, Metrologia 49 (5) (2012) S267.

[31] C. Koch, K.-V. Jenderka, Final report on key comparison CCAUV. U-K3 for ultrasonic power, Metrologia 51 (1A) (2014) 09001.

[32] V. Saptari, Fourier transform spectroscopy instrumentation engineering, Vol. 61, SPIE press, 2004, Ch. 5.

[33] M. Weber, V. Wilkens, Using a heterodyne vibrometer in combination with pulse excitation for primary calibration of ultrasonic hydrophones in amplitude and phase, Metrologia 54 (4) (2017) 432.

[34] C. Koch, W. Molkenstruck, Primary calibration of hydrophones with extended frequency range 1 to $70 \mathrm{MHz}$ using optical interferometry, IEEE Transactions on ultrasonics, ferroelectrics, and frequency control 46 (5) (1999) 1303-1314.

[35] C. Koch, G. Ludwig, W. Molkenstruck, Calibration of a fiber tip ultrasonic sensor up to $50 \mathrm{MHz}$ and the application to shock wave measurement, Ultrasonics 36 (1-5) (1998) 721-725.

[36] B. E. Treeby, B. T. Cox, k-wave: MATLAB toolbox for the simulation and reconstruction of photoacoustic wave fields, Journal of Biomedical Optics 15 (2) (2010) 021314. 
[37] International Organization for Standardization and International Electrotechnical Comission, IEC 17025: General requirements for the competence of testing and calibration laboratories (2017).

[38] B. Karaböce, Y. Gülmez, S. Rajagapol, A. Shaw, Instantaneous input electrical power measurements of HITU transducer, in: Journal of Physics: Conference Series, Vol. 279, IOP Publishing, 2011, p. 012011.

[39] UKAS, United Kingdom Accreditation Service - The expression of uncertainty and confidence in measurement. M3003 - Ed. 3 (2012).

[40] JCGM, 100:2008 Evaluation of measurement data - Guide to the expression of uncertainty in measurement (GUM 1995 with minor corrections) (2008).

[41] R. M. Baêsso, R. P. Costa-Felix, P. Miloro, B. Zeqiri, Ultrasonic parameter measurement as a means of assessing the quality of biodiesel production, Fuel 241 (2019) 155-163.

[42] R. A. Johnson, D. W. Wichern, et al., Applied multivariate statistical analysis, Vol. 5, Prentice Hall Upper Saddle River, NJ, 2002.

[43] IEEE, 178-1958: IEEE Standards on Piezoelectric Crystals:Determination of The Elastic. Piezoelectric and Dielectric Constants - The Electro mechanical Coupling Factor (1958).

[44] BIMP, Calibration and measurement capabilities in the context of the cipm mra, https://www.bipm.org/kcdb/cmc/quick-search, accessed: 2020$12-01$.

[45] Precision Acoustics Ltd, Radiation force balance, https://www. acoustics.co.uk/product/radiation-force-balance/, accessed: 202012-01.

[46] S. Maruvada, G. R. Harris, B. A. Herman, R. L. King, Acoustic power calibration of high-intensity focused ultrasound transducers using a radiation 
force technique, The Journal of the Acoustical Society of America 121 (3) (2007) 1434-1439.

720

[47] A. Shaw, A buoyancy method for the measurement of total ultrasound power generated by HIFU transducers, Ultrasound in Medicine \& Biology 34 (8) (2008) 1327-1342.

[48] R. A. Smith, D. R. Bacon, A multiple-frequency hydrophone calibration technique, The Journal of the Acoustical Society of America 87 (5) (1990) 2231-2243. 